\title{
Retours du congrès EuroMedLab 2019
}

\author{
Laurence Piéroni ${ }^{1}$ \\ Vincent Sapin ${ }^{2}$ \\ ${ }^{1}$ Rédactrice en chef des $\mathrm{ABC}$ \\ $<$-pieroni@chu-montpellier.fr> \\ 2 Président de la SFBC
}

Cette année 2019, Barcelone a accueilli le $23^{\text {e }}$ congrès EuroMedLab, manifestation scientifique organisée tous les deux ans par l'IFCC et l'EFLM.

À cette occasion, la SFBC a sollicité ses membres représentant la France au sein de ces organisations internationales, afin qu'ils transmettent à leur retour une partie de leur expérience espagnole aux lecteurs des ABC.

Par ailleurs, la SFBC a financé le déplacement d'internes, grâce à l' attribution de bourses, afin de faciliter la promotion de leur travail de recherche. Leur contribution est également présentée dans ce numéro des $\mathrm{ABC}$.

Bruno Baudin, Guilaine Boursier, Vincent Delatour, Philippe Gillery, Bernard Gouget, Damien Gruson, Laurence
Piéroni, Michel Vaubourdolle, d'une part, et Aurore Desmous, Emma Galofaro, Mohamed Chtourou, Olivier Grunewald, d'autre part, se sont prêtés au jeu et vous font partager une sélection des conférences auxquelles ils ont pris part.

Les auteurs ayant eu le choix du format et du sujet, les articles sont donc hétérogènes mais tout aussi intéressants les uns que les autres et vous motiveront probablement pour participer au prochain congrès EuroMedLab, prévu en 2021 à Munich.

Liens d'intérêts : les auteurs déclarent ne pas avoir de lien d'intérêts en rapport avec cet article. 